\title{
DIFFERENTIAL GAMES WITH UNCERTAIN TERMINAL TIME
}

\author{
Ilan Rusnak, Gyorgy Hexner and Haim Weiss
}

RAFAEL, P. O. Box 2250, Haifa ISRAEL 31021

\begin{abstract}
Linear Quadratic Differential games have been used to model economic and other conflict situations. A salient feature of these analyses was that the interval of play was fixed (possibly inifinite), and known to both players. This paper introduces a linear quadratic differential game where only the probability density of the terminal time is known to the two players. The paper's results are used to derive a differential game based guidance law for uncertain time of intercept. Copyright @ 2005 IFAC
\end{abstract}

Keywords: Differential game, Linear quadratic, Uncertain terminal time

\section{INTRODUCTION}

Since its introduction in (Ho et al., 1965), and in (Bryson and Ho, 1969) linear differential games have been used in various situations. Further properties of linear quadratic differential games were developed in (Bernhard, 1979) and (Mageirou, 1976). The purpose of this paper is to introduce a linear quadratic differential game where the terminal time is unknown by the two players. Each of the players knows only the probability density of the terminal time.

There are two features of the terminal time uncertainty that are explicitly assumed here:

- Both players are initially supplied with the probability density function of the terminal time.

- The players derive no additional information about the terminal time from their observations.

The first treatment of a linear quadratic problem with an uncertain terminal time was in (Sivan, 1966). In (Rusnak, 2004) a one sided linear quadratic optimization problem was solved and applied to missile terminal guidance. Previous to (Rusnak, 2004) it had been known that modern guidance laws were sensitive to errors in terminal time, (Nesline and Zarchan, 1981). A modern guidance law with reduced sensitivity to uncertainty in terminal time was suggested in (BenAsher and Yaesh, 1998).

The remainder of this paper is organized as follows: In the next section, the problem is stated. Then the solution of the problem is presented. This is followed by the the application of these results to the terminal guidance problem.

\section{PROBLEM STATEMENT}

The differential game is played by two players, a pursuer and an evader, denoted as $\mathrm{P}$ and $\mathrm{E}$. $\mathrm{P}$ selects the control variable $\mathbf{u}$ and $\mathrm{E}$ selects the control variable $\mathbf{v}$. They jointly control the dynamic system

$$
\dot{\mathbf{x}}=\mathbf{A x}+\mathbf{B}_{\mathbf{u}} \mathbf{u}+\mathbf{B}_{\mathbf{v}} \mathbf{v} .
$$

Player P's aim is to minimize and player E's aim is to maximize the expected value of the cost function $J(T)$, 


$$
\begin{gathered}
J(T)=\frac{1}{2} \mathbf{x}(T)^{T} \mathbf{G}(T) \mathbf{x}(T)+ \\
\frac{1}{2} \int_{0}^{T}\left(\mathbf{x}^{T} \mathbf{Q} \mathbf{x}+\mathbf{u}^{T} \mathbf{R}_{\mathbf{u}} \mathbf{u}-\mathbf{v}^{T} \mathbf{R}_{\mathbf{v}} \mathbf{v}\right) d s
\end{gathered}
$$

Neither player knows the terminal time, $T$, but both players are aware of a common probability density, $p(T)$ of the terminal time. It is assumed that the matrices, $\mathbf{Q}, \mathbf{R}_{\mathbf{u}}$, and $\mathbf{R}_{\mathbf{v}}$ are defined for all values of the terminal time, $T$ such that $p(T)>0$.

\section{THE SOLUTION}

The basic method of the solution is to explicitly calculate the expected value of the criterion function $J$, and observe that after the calculation the problem is equivalent to a differential game played over a fixed (possibly infinite) interval.

\subsection{The details of the solution}

The expected value of $J$ is,

$$
\begin{gathered}
E\{J\}=\frac{1}{2} \int_{0}^{\infty} \mathbf{x}(T)^{T} \mathbf{G} \mathbf{x}(T) p(T) d T+ \\
\frac{1}{2} \int_{0}^{\infty} \int_{0}^{T}\left(\mathbf{x}^{T} \mathbf{Q} \mathbf{x}+\mathbf{u}^{T} \mathbf{R}_{\mathbf{u}} \mathbf{u}-\mathbf{v}^{T} \mathbf{R}_{\mathbf{v}} \mathbf{v}\right) p(T) d s d T
\end{gathered}
$$

Interchanging the order of the integration the expression for $E\{J\}$ becomes,

$$
\begin{gathered}
E\{J\}=\frac{1}{2} \int_{0}^{\infty} \mathbf{x}(T)^{T} \mathbf{G} \mathbf{x}(T) p(T) d T+ \\
\frac{1}{2} \int_{0}^{\infty} \int_{s}^{\infty}\left(\mathbf{x}^{T} \mathbf{Q} \mathbf{x}+\mathbf{u}^{T} \mathbf{R}_{\mathbf{u}} \mathbf{u}-\mathbf{v}^{T} \mathbf{R}_{\mathbf{v}} \mathbf{v}\right) p(T) d T d s
\end{gathered}
$$

Define the following quantities:

$$
\begin{aligned}
\boldsymbol{\Theta}(t) & =\underset{\mathbf{G} p(t),}{\boldsymbol{\Psi}_{\mathbf{u}}(t)}=\int_{t}^{\infty} \mathbf{R}_{\mathbf{u}} p(s) d s, \\
\boldsymbol{\Psi}_{\mathbf{v}}(t) & =\int_{t}^{\infty} \mathbf{R}_{\mathbf{v}} p(s) d s, \\
\mathbf{\Phi}(t) & =\int_{t}^{\infty} \mathbf{Q} p(s) d s .
\end{aligned}
$$

Then $E\{J\}$ may be expressed as

$$
\begin{gathered}
E\{J\}= \\
\frac{1}{2} \int_{0}^{\infty}\left(\mathbf{x}^{T}(\boldsymbol{\Theta}+\boldsymbol{\Phi}) \mathbf{x}+\mathbf{u}^{T} \boldsymbol{\Psi}_{\mathbf{u}} \mathbf{u}-\mathbf{v}^{T} \boldsymbol{\Psi}_{\mathbf{v}} \mathbf{v}\right) d s
\end{gathered}
$$

Equation (6) is just the cost function for a standard linear-quadratic differential game. It's solution, and properties are then the same as that of a standard linear quadratic differential game. In particular the differential game has a well defined solution provided the Riccati differential equation,

$$
\begin{gathered}
-\dot{\mathbf{P}}=\mathbf{P A}+\mathbf{A}^{T} \mathbf{P}-(\boldsymbol{\Theta}+\boldsymbol{\Phi}) \\
+\mathbf{P}\left(\mathbf{B}_{\mathbf{u}} \boldsymbol{\Psi}_{\mathbf{u}}{ }^{-1} \mathbf{B}_{\mathbf{u}}{ }^{T}-\mathbf{B}_{\mathbf{v}} \boldsymbol{\Psi}_{\mathbf{v}}{ }^{-1} \mathbf{B}_{\mathbf{v}}{ }^{T}\right) \mathbf{P}
\end{gathered}
$$

with initial condition $\mathbf{P}\left(T_{f}\right)=\mathbf{0}$, where $T_{f}$ is a time such that $p(t)=0, \forall t>T_{f}$. The two players' optimal strategies are

$$
u=-\mathbf{B}_{\mathbf{u}}{ }^{T} \Psi_{\mathbf{u}}{ }^{-1} \mathbf{P} \mathbf{x}
$$

and

$$
v=\mathbf{B}_{\mathbf{v}}^{T} \Psi_{\mathbf{v}}^{-1} \mathbf{P} \mathbf{x}
$$

\section{APPLICATION TO GUIDANCE}

The underlying model used for studying the guidance laws in the face of uncertainty in the terminal time is the same one used in (Bryson and Ho, 1969) and (Ho et al., 1965). It is assumed that the pursuer and its target are near collision course. $y$ and $\dot{y}$ are the relative position and velocity of the evader and the pursuer perpendicular to the initial line sight. Then

$$
\left[\begin{array}{c}
\dot{y} \\
\dot{y}
\end{array}\right]=\left[\begin{array}{ll}
0 & 1 \\
0 & 0
\end{array}\right]\left[\begin{array}{l}
y \\
\dot{y}
\end{array}\right]+\left[\begin{array}{l}
0 \\
1
\end{array}\right] u+\left[\begin{array}{c}
0 \\
-1
\end{array}\right] v
$$

and

$$
G(T)=\left[\begin{array}{cc}
g_{f} & 0 \\
0 & 0
\end{array}\right]
$$

where $g_{f}$ is the weight coefficient of the quadratic term in the miss distance.

The feedback law here is a linear combination of the distance and velocity $y$ and $\dot{y}$ perpendicular to the initial line of sight. In the case that there is no uncertainty in the terminal time it was shown in (Bryson and Ho, 1969) that the guidance law obtained is in fact proportional navigation, which depends only on the line of sight inertial angular rate. However, even if there is a small uncertainty in the terminal time this is no longer the case. Nevertheless in order to compare the present results to the classical proportional navigation it is desired to express the feedback law in terms of the line of sight angle and angular rate. For this purpose it is assumed that the extent of the uncertainty in the terminal time is small with respect to the initial interval. 


\subsection{Approximations for Small Uncertainty in Time to go}

For the remainder of the paper it is assumed that the closing velocity, $V_{c}$ may be considered constant during the time interval of the differential game. Then in general the line of sight angle, $\lambda$ is

$$
\lambda=\frac{y}{V_{c} t_{g}},
$$

where $t_{g}$ is the time until the termination of the game. Expanding $\lambda$ to first order in $t_{g}$ and using the remainder theorem it is noted that

$$
\lambda=\lambda\left(t_{g 0}\right)-\left(\frac{y}{t_{g 1}^{2} V_{c}}\right) \Delta t_{g},
$$

where $t_{g 1}$ is a point within the time to go interval uncertainty. But this may be rewritten as

$$
\lambda=\lambda\left(t_{t 0}\right)-\lambda\left(t_{g 1}\right) \frac{\Delta t_{g}}{t_{g 1}}
$$

Therefore it may be concluded that as long as $\frac{\Delta t_{g}}{t_{g}} \ll 1$ the error in the approximating $\lambda$ by equation(12), within the uncertainty region of $t_{g}$ is small.

A similar bound is required in approximating the inertial line of sight rate, $\dot{\lambda}$. This is obtained by differentiating equation(12),

$$
\dot{\lambda}=\frac{1}{V_{c}}\left(y t_{g}^{-2}+\dot{y} t_{g}^{-1}\right)
$$

Expanding $\dot{\lambda}$ to first order in $t_{g}$ and using the remainder theorem,

$$
\dot{\lambda}\left(t_{g}\right)=\dot{\lambda}\left(t_{g 0}\right)+\frac{1}{V_{c}}\left(-2 t_{g 1}^{-3}-\dot{y} t_{g 1}^{-2}\right) \Delta t_{g}
$$

where $t_{g 1}$ is a point within the time to go interval of uncertainty. But equation(16) may be rewritten as

$$
\dot{\lambda}=\dot{\lambda}\left(t_{g 0}\right)-\left(\dot{\lambda}_{1}+\frac{\lambda_{1}}{t_{g 1}}\right) \frac{\Delta t_{g}}{t_{g 1}}
$$

Therefore, it may be concluded that as long as

$$
\frac{\Delta t_{g}}{t_{g}} \ll 1
$$

$\lambda$ and $\dot{\lambda}$ may be approximated with small error by using a single time point, $t_{g}$ within the time to go uncertainty region.

\subsection{The Guidance Law in Terms of $\lambda$ and $\dot{\lambda}$}

In order to study the influence of the time to go uncertainty on the optimal guidance law it is necessary to express the guidance law in terms of the line of sight angle, $\lambda$ and the line of sight angular rate, $\dot{\lambda}$. For this purpose the validity of inequality(18) is assumed. The guidance law in equations (8) and (9) is specialized for equation (10). The pursuer control law may be expressed as

$$
u=k_{y}^{(u)} y+k_{\dot{y}}^{(u)} \dot{y}
$$

and that for the evader,

$$
v=k_{y}^{(v)} y+k_{\dot{y}}^{(v)} \dot{y}
$$

The state variables $y$ and $\dot{y}$ may be expressed in terms of the line of sight angle and line of sight angular rate, $\lambda$ and $\dot{\lambda}$ as,

$$
y=V_{c} t_{g} \lambda
$$

and

$$
\dot{y}=V_{c} t_{g} \dot{\lambda}-V_{c} \lambda
$$

In terms of $\lambda$ and $\dot{\lambda}$ the guidance law is

$$
u=k_{\lambda}^{(u)} \lambda+k_{\dot{\lambda}}^{(u)} \dot{\lambda}
$$

and

$$
v=k_{\lambda}^{(v)} \lambda+k_{\dot{\lambda}}^{(v)} \dot{\lambda}
$$

where

$$
\begin{gathered}
k_{\lambda}^{(u)}=V_{c}\left(k_{y}^{(u)} t_{g}-k_{\dot{y}}^{(u)}\right) \\
k_{\dot{\lambda}}^{(u)}=V_{c} t_{g} k_{\dot{y}}^{(u)} \\
k_{\lambda}^{(v)}=V_{c}\left(k_{y}^{(v)} t_{g}-k_{\dot{y}}^{(v)}\right) \\
k_{\dot{\lambda}}^{(v)}=V_{c} t_{g} k_{\dot{y}}^{(v)}
\end{gathered}
$$

\subsection{Examples}

In this section the effect of the final time uncertainty on the guidance problem presented in this section is studied. In all the cases the following parameters are used:

$$
\begin{aligned}
g_{f} & =10^{12} \\
R_{u} & =1 \\
R_{v} & =2 \\
\mathbf{Q} & =\mathbf{0} \\
T_{f} & =2 \mathrm{sec}
\end{aligned}
$$

In all the following examples the discrete Riccati equation equivalent to the continuous time Riccati differential equation(7) was solved with a sampling time of $1 \mathrm{mSec}$. 
4.3.1. The Classical Differential Game Guidance Law In this first example there is no uncertainty in the terminal time,

$$
p(T)=\delta\left(T-T_{f}\right)
$$

If in addition we let $g_{f} \rightarrow \infty$, then the solution is simply the well known classical differential game law:

$$
\begin{gathered}
k_{\lambda}^{(u)}=0 \\
k_{\lambda}^{(v)}=0 \\
k_{\dot{\lambda}}^{(u)}=\frac{3 V_{c}}{1-\frac{R_{u}}{R_{v}}} \\
k_{\dot{\lambda}}^{(v)}=\frac{3 V_{c}}{\frac{R_{v}}{R_{u}}-1}
\end{gathered}
$$

A more common description of a guidance law is the navigation constant $N^{\prime}$, which is,

$$
N^{(\cdot) \prime}=\frac{1}{V_{c}} k_{\dot{\lambda}}^{(\cdot)}
$$

and the gain

$$
N_{\lambda}^{(\cdot)}=\frac{t_{g}}{N^{(\cdot) /} V_{c}} k_{\lambda}^{(\cdot)}
$$

In terms of $N^{\prime}$ and $N_{\lambda}$ the command $u$ is

$$
u=N^{\prime} V_{c}\left(\dot{\lambda}+N_{\lambda} \frac{\lambda}{t_{g}}\right)
$$

In this case, where there is no uncertainty in the terminal time, the gains $N^{\prime}$ and $N_{\lambda}$ are plotted in figure(1). The upper plot shows the navigation ratio $\left(N^{\prime}\right)$ for the pursuer: dotted line; and, for the evader: full line. The lower plot shows the the gain $N_{\lambda}$, (in this case $N_{\lambda}=0$ ).

4.3.2. Uniform Uncertainty of $10 \mathrm{mS}$ The present and the following examples model the effects of a $(10 \mathrm{mS})$ uncertainty on the optimal guidance gains. When the probability density of the terminal time is uniformly distributed over a $10 \mathrm{mS}$ time interval, then the gains shown in figure(2) are obtained. The graphs plotted have the same description as in figure(1): The upper plot shows the navigation ratio $\left(N^{\prime}\right)$ for the pursuer: dotted line; and, for the evader: full line. The lower plot shows the gain $N_{\lambda}$. Note that even such a small uncertainty has a very substantial effect on the guidance gains. In particular there is now a nonzero gain for the $N_{\lambda}$ term.

4.3.3. Discrete Uncertainty of $10 \mathrm{mS}$ In the case at hand the probability density of the terminal time is

$$
p(T)=\frac{1}{2} \delta\left(T-T_{f}\right)+\frac{1}{2} \delta\left(T-T_{f}-0.01\right)
$$
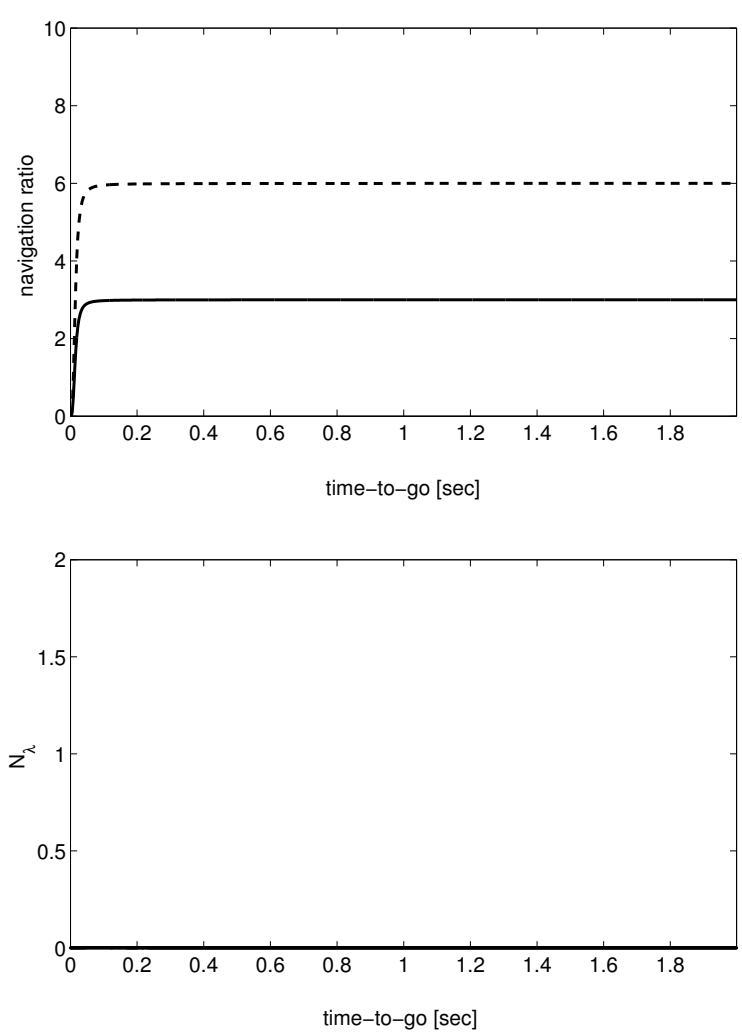

Fig. 1. The guidance gains for the classical differential game.
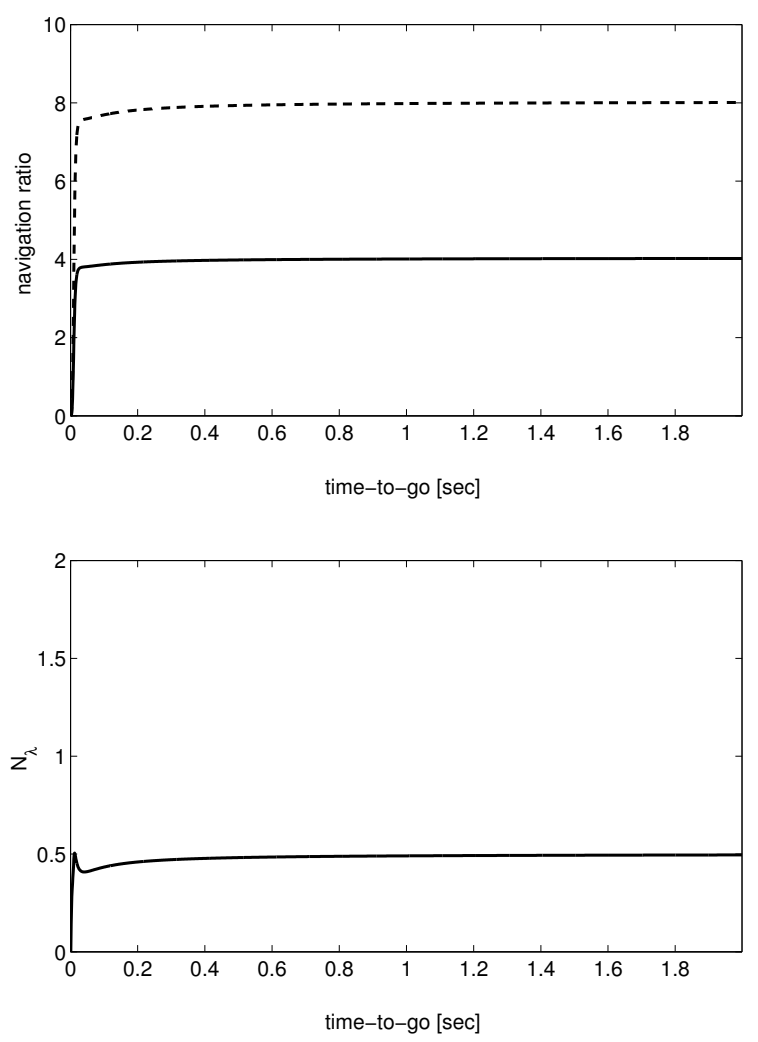

Fig. 2. The guidance gains for uniform uncertainty of $10 \mathrm{mSec}$. 

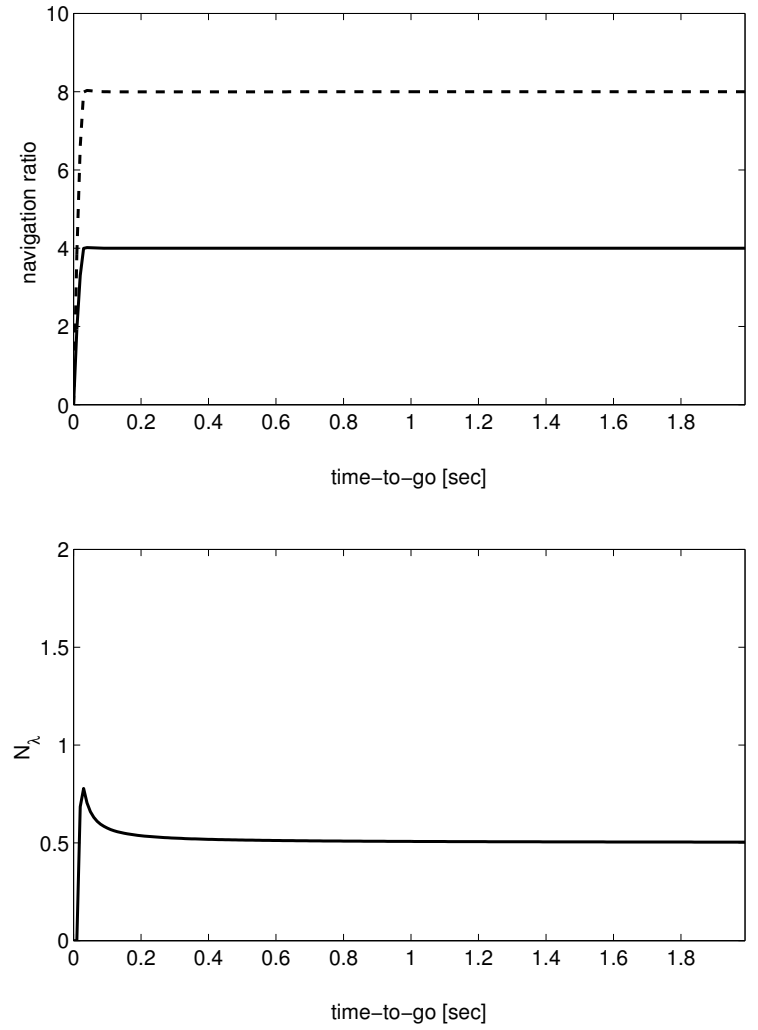

Fig. 3. The guidance gains for discrete uncertainty.

The gains for this case are presented in figure(3). The graphs plotted have the same description as in figure(1): The upper plot shows the navigation ratio $\left(N^{\prime}\right)$ for the pursuer: dotted line; and, for the evader: full line. The lower plot shows the gain $N_{\lambda}$.

4.3.4. Non-symetric Uncertainty In the development in section (3) it is not necessary to assume that the two players' uncertainty probability densities are identical. In fact a very similar development can be made for the case that the two players have different probability densities for the termination time of the game. The details are presented in the Appendix.

In the guidance problem if each player is aware of the other's information structure, for example if each knows the additive noise of his own as well as his opponent's observation noise, then each player is able to calculate his own as well as his opponent's probability density, and hence both his own and his opponent's cost function.

In the present scenario the minimizing player's probability density is

$$
p_{u}(T)=\frac{1}{2} \delta\left(T-T_{f}\right)+\frac{1}{2} \delta\left(T-T_{f}-0.1\right)
$$

and the probability density of the maximizing player is

$$
p_{v}(T)=\frac{1}{2} \delta\left(T-T_{f}\right)+\frac{1}{2} \delta\left(T-T_{f}-0.2\right)
$$
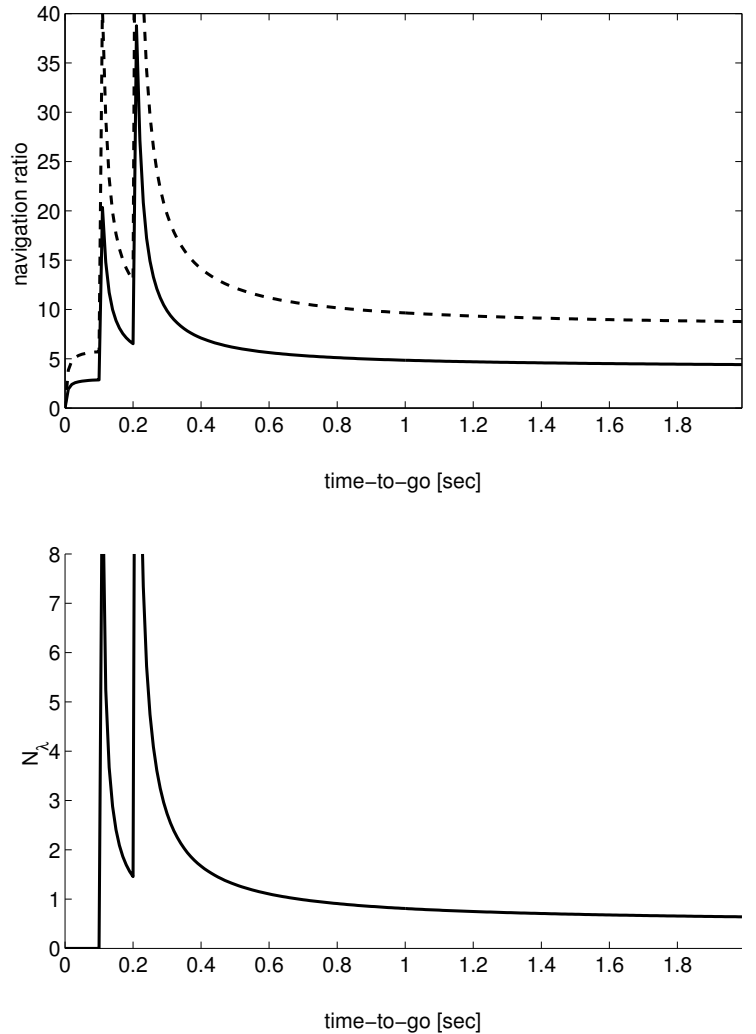

Fig. 4. The guidance gains for non-symetric uncertainty.

The following values for the parameters in the Appendix were used in the example:

$$
\begin{gathered}
\mathbf{G}_{\mathbf{u}}=\mathbf{G}_{\mathbf{v}}=\left[\begin{array}{cc}
10^{12} & 0 \\
0 & 0
\end{array}\right] \\
\mathbf{Q}_{\mathbf{u}}=\mathbf{Q}_{\mathbf{v}}=\mathbf{0} \\
\mathbf{R}_{\mathbf{u}}=1, \mathbf{R}_{\mathbf{v}}=2 \\
T_{f}=2 \mathrm{sec}
\end{gathered}
$$

The guidance gains are presented in figure(4). The approximation condition equation(18) restricts the validity of the approximation of the line of sight angle and angular rate equations(12) and (15) to the first few tenths of a second from the start of the game. Note, however that this validity condition applies to the gain $N^{(\cdot) \prime}$ and $N_{\lambda}^{(\cdot)}$ calculations only, the gains $k_{\dot{y}}^{(\cdot)}$ and $k_{y}^{(\cdot)}$ are valid regardless of the approximation in inequality(18). The graphs plotted have the same description as in figure(1): The upper plot shows the navigation ratio $\left(N^{\prime}\right)$ for the pursuer: dotted line; and, for the evader: full line. The lower plot shows the gain $N_{\lambda}$.

\subsection{Discussion}

The most surprising feature of the guidance laws developed here is the large change that they undergo for even a small $(10 \mathrm{mSec})$ uncertainty in the terminal time. The main change is that the 
guidance law depends on $\lambda$ as well as on $\dot{\lambda}$. In fact the guidance laws have the form of the well known "rendez vous" guidance law:

$$
u=4 V_{c}\left(\dot{\lambda}+\frac{1}{2} \frac{\lambda}{t_{g}}\right)
$$

which is obtained by setting

$$
G(T)=\left[\begin{array}{cc}
g_{f} & 0 \\
0 & \bar{g}_{f}
\end{array}\right]
$$

in equation (11), and letting both $g_{f}$ and $\bar{g}_{f}$ tend to infinity. That is, the pursuer attempts to cause the intercept to occur along the line of sight, while the evader tries to counter and to drive the intercept out of this course. The miss results from the uncertainty in the intercept time multiplied by the the relative velocity perpendicular to the line of sight. This explains the results in (Ben-Asher and Yaesh, 1998) and (Rusnak, 2004).

Obseve that $N_{\lambda}$ in figures (2), (3), and (4) is $1 / 2$ for $t_{g} \gg 0$. This is the same value that appears in equation(40).

\section{SUMMARY}

This paper introduced linear quadratic differential games with uncertain terminal time. An explicit formula for the solution of this class of differential games was presented. The results were applied to a guidance problem in a differential game setting. It was shown that even a very small uncertainty in the intercept time profoundly influences the guidance law.

\section{Appendix A. NON-SYMETRIC UNCERTAINTY}

In the more general case where each has a probability density for the terminal time, the cost function is

$$
\begin{aligned}
& J=\frac{1}{2} \mathbf{x}\left(T_{u}\right)^{T} \mathbf{G}_{\mathbf{u}} \mathbf{x}\left(T_{u}\right)+ \\
& \frac{1}{2} \int_{0}^{T_{u}}\left(\mathbf{x}^{T} \mathbf{Q}_{\mathbf{u}} \mathbf{x}+\mathbf{u}^{\mathbf{T}} \mathbf{R}_{\mathbf{u}} \mathbf{u}\right) d s+ \\
& \begin{array}{c}
\frac{1}{2} \mathbf{x}\left(T_{v}\right) \mathbf{G}_{\mathbf{v}} \mathbf{x}\left(T_{v}\right)+ \\
\frac{1}{2} \int_{0}^{T_{v}}\left(\mathbf{x}^{T} \mathbf{Q}_{\mathbf{v}} \mathbf{x}-\mathbf{v}^{T} \mathbf{R}_{\mathbf{u}} \mathbf{v}\right) d s
\end{array}
\end{aligned}
$$

The expected value of $J$ is given by,

$$
\mathbf{E}(J)=\int_{0}^{\infty} \int_{0}^{\infty} J p_{u}\left(T_{u}\right) p_{v}\left(T_{v}\right) d T_{u} d T_{v}
$$

where $p_{u}\left(T_{u}\right)$ and $p_{v}\left(T_{v}\right)$ are the probability density functions of the termination times for the two players. The derivation is similar to that described in Section(3), but with the symbols $\boldsymbol{\Theta}, \boldsymbol{\Psi}_{\mathbf{u}}, \boldsymbol{\Psi}_{\mathbf{v}}$, and $\boldsymbol{\Phi}$ in equation(5) replaced by

$$
\begin{aligned}
\boldsymbol{\Theta}(t) & =\mathbf{G}_{\mathbf{u}} p_{u}(t)+\mathbf{G}_{\mathbf{v}} p_{v}(t) \\
\boldsymbol{\Psi}_{\mathbf{u}}(t) & =\int_{t}^{\infty} \mathbf{R}_{\mathbf{u}} p_{u}(s) d s \\
\boldsymbol{\Psi}_{\mathbf{v}}(t) & =\int_{t}^{\infty} \mathbf{R}_{\mathbf{v}} p_{v}(s) d s \\
\mathbf{\Phi}(t) & =\int_{t}^{\infty} \mathbf{Q}_{\mathbf{u}} p_{u}(s) d s+\int_{t}^{\infty} \mathbf{Q}_{\mathbf{v}} p_{v}(s) d s
\end{aligned}
$$

Once the substitutions are made, the Riccati differential equation(7), and the control strategies for the two players, equations(8) and (9) remain unchanged.

Note that the problem described in Section(2) is obtained by setting

$$
\begin{gathered}
p(\cdot)=p_{u}(\cdot)=p_{v}(\cdot) \\
\mathbf{G}=\mathbf{G}_{\mathbf{u}}+\mathbf{G}_{\mathbf{v}} \\
\mathbf{Q}=\mathbf{Q}_{\mathbf{u}}+\mathbf{Q}_{\mathbf{v}}
\end{gathered}
$$

\section{REFERENCES}

Ben-Asher, J. and I. Yaesh (1998). Advances in Missile Guidance Theory, Progress in Astronautics and Aeronautics, Vol. 180. AIAA Inc.. Washington, D. C.

Bernhard, P. (1979). Linear quadratic, two person zero sum differential games; necessary and sufficient conditions. Journal of Otimization Theory and Applications 27, 51-69.

Bryson, A. E. and Y. C. Ho (1969). Applied Optimal Control. Blaisdel Publishing Company. New York.

Ho, Y. C., A. E. Bryson and S. Baron (1965). Differential games and optimal pursuit evasion strategies. IEEE Transactions on Automatic Control AC-10, 385-389.

Mageirou, E. F. (1976). Values and strategies for infinite time linear quadratic games. IEEE Transaction on Automatic Control AC-21, 547-550.

Nesline, F. W. and P. Zarchan (1981). A new look at classical versus modern homing guidance. Journal of Guidance, Control and Dynamics 4, 78-85.

Rusnak, I. (2004). Optimal guidance laws with uncertain time-of-flight. IEEE Transactions on Aerospace and Electronic Systems AES23, 742-746.

Sivan, R. (1966). The optimal control of linear systems with unknown final time. IEEE Transactions on Automatic Control AC-11, 529530. 